\title{
Computing Morse Decompositions for Triangulated Terrains: An Analysis and an Experimental Evaluation
}

\author{
Maria Vitali, Leila De Floriani, and Paola Magillo \\ University of Genova, Italy \\ \{vitali, deflo,magillo\}@disi.unige.it
}

\begin{abstract}
We consider the problem of extracting the morphology of a terrain discretized as a triangle mesh. We discuss first how to transpose Morse theory to the discrete case in order to describe the morphology of triangulated terrains. We review algorithms for computing Morse decompositions, that we have adapted and implemented for triangulated terrains. We compare the the Morse decompositions produced by them, by considering two different metrics.
\end{abstract}

\section{Introduction}

Modeling the morphology of a real terrain is a relevant issue in several application domains, including terrain analysis and understanding, knowledge-based reasoning, and hydrological simulation. Morphology consists of feature points (pits, peaks and passes), feature lines (like ridges and ravines), or segmentation of the terrain in regions of influence of minima and maxima or in regions of uniform gradient field. Morphological models of terrains are rooted in Morse theory 26. Based on Morse theory cellular decompositions of the graph of a scalar field defined on a manifold, called Morse complexes, are defined by considering the critical points of the field and the integral lines. The same mathematical and algorithmic tools apply for segmenting 3D shapes based on a scalar field defined over the shape, such as curvature [22 23/24]. Unfortunately, the results from Morse theory apply only to smooth functions, while the most common terrain and shape models are piece-wise linear functions defined over a triangle mesh. Current approaches [46] try to simulate Morse complexes in the discrete case, but pose constraints (i.e., flat edges are not allowed), that are usually not satisfied by real terrains or $3 \mathrm{D}$ shapes.

The purpose of this work is to review, analyze and compare algorithms for computing Morse complexes on triangulated surfaces built on real data sets. We focus our attention on terrains modeled as piece-wise linear triangulated surfaces with vertices at the sample points, called Triangulated Irregular Networks (TINs). First, we discuss how to overcome the limitations of current discrete approaches by dealing with flat edges effectively. We classify flat edges and then discuss how to eliminate singularities from TINs through a process of edge collapse which may generate new critical points. 
In the literature, two major approaches exist for computing Morse decompositions of discrete terrain models: region-based algorithms compute the 2-cells of the Morse complexes while boundary-based algorithms compute the lines forming their boundaries. We compare results on real datasets, and we consider both noisy data and data after a smoothing process and elimination of the singularities. We compare the segmentations produced by the various algorithms using two metrics.

The remainder of the paper is organized as follows. In Section 2, we present background notions on Morse theory. In Section 3, we present discrete approaches to Morse theory and our new characterization of Morse functions in the discrete, that we call extended Piecewise Linear (ePL)-Morse functions. In Section 4 , we review algorithms to decompose a terrain into Morse complexes. In Section 5 we discuss the metrics, used in Section 6 to compare the Morse decompositions produced by the different algorithms. Finally, in Section 7 , we draw some concluding remarks, and discuss future developments.

\section{Background Notions}

Let us consider a domain $M$ in the three-dimensional Euclidean space and a smooth real-valued function $f$ defined over $M$. A point $p$ of $M$ is a critical point of $f$ if the gradient of $f$ at $p$ is null. Critical points of $f$ are minima, saddles and maxima. All other points are called regular. A critical point $p$ is degenerate if the determinant of the Hessian matrix of $f$ at $p$ is zero. A function $f$ is called a Morse function [26], if all its critical points are not degenerate. A Morse function admits only finitely many isolated critical points.

An integral line of $f$ is a maximal path which is everywhere tangent to the gradient of $f$. Integral lines that converge to a minimum [saddle, maximum] $p$ form a 0-cell [1-cell, 2-cell] called the stable cell of $p$. Similarly, integral lines that originate from a minimum [saddles, maximum] $p$ form a 2-cell [1-cell, 0-cell] called the unstable cell of $p$. The stable (unstable) cells decompose $M$ into a Euclidean cell complex, called a stable (unstable) Morse complex. If the unstable and the stable cells intersect transversally, function $f$ is said to satisfy the Smale condition. If $f$ satisfies the Smale condition, the overlay of stable and unstable Morse complexes is called a Morse-Smale complex.

A Morse complex is also related with the concept of watershed transform [29], which provides a decomposition of the domain of a smooth function $f$ into open regions of influence associated with the minima of $f$, called catchment basins. Catchment basins correspond to the cells of the stable Morse complex defined on the same domain. The regions of influence related to the saddles form the watershed lines.

\section{Discrete Approaches to Morse Theory}

In the literature there are two extensions of Morse Theory to a discrete domain. The discrete Morse theory proposed by Forman [18] presents an adaptation of 
Morse theory that can be applied to any simplicial or cell complex. Forman assumes to have a function value associated with each cell of the complex (not just the vertices). Thus, it is not directly useful in our case. The piecewise linear Morse theory by Banchoff [4] extends Morse theory to piecewise-linear functions defined on triangulated surfaces, under the assumption is that every pair of points of the triangulated surface have distinct field values. In order to define the conditions for a vertex $v$ to be critical, the polyhedral surface made by the triangles incident in $v$ is considered, and the number $I$ of intersections between such surface and the plane parallel to the $x$ - $y$-plane, which is passing through $v$, is counted. If there are no intersections, $v$ is a maximum or a minimum. If there are two intersections, $v$ is a regular vertex. Otherwise $v$ is a saddle: a simple saddle if $I=4$, or a multiple saddle if $I>4$. Note that $I$ is always even. The multiplicity of a saddle is equal $I / 2$.

Banchoff's assumption on piece-wise linear Morse functions is quite strong, and thus it is often replaced by the weaker condition that each pair of adjacent vertices (i.e., connected by an edge) have different elevation values [6]. Such condition ensures a decomposition of the domain, which has the same properties as a Morse complex, called a Piece-wise Linear Morse Complex (PLMC). The above condition is still too strong for real data sets. In 15, we have defined a classification of an isolated flat edge $e$ based on the value $f_{e}$ of function $f$ on $e$, and on the values $f_{C}$ and $f_{D}$ of $f$ at the other vertices of the triangles incident into $e$. If $f_{C}<f_{e}<f_{D}$ or $f_{D}<f_{e}<f_{C}$, then $e$ is a regular edge. If $f_{e}<\min \left(f_{C}, f_{D}\right)$ or $f_{e}>\max \left(f_{C}, f_{D}\right)$ then $e$ is a critical edge. We say that the elevation function defined at the vertices of a TIN is an extended PL (ePL)Morse function if it does not contain critical edges or flat triangles. TINs that present only isolated flat edges can be reduced to TINs for ePL-Morse functions by collapsing such edges.

\section{Algorithms for Computing Morse Decompositions}

In the literature several approaches have been proposed for providing an approximation of the Morse and Morse-Smale complexes in the discrete.

Boundary-based algorithms (see Section 4.1) extract the Morse-Smale complex by tracing the integral lines, or their approximations, starting from saddle points and converging to minima and maxima. Region based algorithms compute the 2-cells of the Morse complexes by growing the neighborhood of minima and maxima (see Section 4.2). Watershed algorithms compute the Morse complexes based on the discrete watershed transform (see Section 4.3).

\subsection{Boundary-Based Algorithms}

Boundary-based algorithms [31/3817/27/31] compute the 2-cells of the MorseSmale complex on a TIN indirectly by computing the 1-cells, i.e., the boundaries of the 2-cells. They first extract the critical points, and then trace the separatrix lines, that are the 1-cells of the Morse-Smale complex. 
The basic idea is due to Takahashi et al [31]. According to Banchoff [4], critical vertices are identified by considering the incident triangles in them. Let us consider the radially sorted list of neighbors of a saddle $s$. Within such sorted list, we consider the maximal sublists formed by vertices with higher (lower) value than $s$ and call them upper (lower) sequences. Starting from each saddle $p$, with multiplicity $k, 2 k$ path are computed. Given $p$, we consider the upper sequences and the lower sequences. We select the first point of each path by choosing the highest (lowest) vertex from each upper (lower) sequence. Then, the ascending (descending) paths are computed by choosing, at each step, the highest (lowest) adjacent vertex until a critical point is reached. Saddles play a key role in this algorithm.

Edelsbrunner et al. 17/6 extend the approach by Takahashi et al. 31. Function $f$ is required to be a piecewise linear Morse function, according to Banchoff's definition, i.e., vertices must have distinct function values. Likewise, ridges and valleys are computed by starting from saddles, but at each step the point with the maximum slope is selected. The Smale condition is simulated a-posteriori by extending the path beyond each saddle, and forcing the path of the critical net not to intersect. The technique is rather complex, it requires edge duplications, and can lead to degenerated 2-cells (see [17] for more details).

\subsection{Region-Growing Algorithms}

Region growing algorithms 9 1220] mimic, in the discrete case, the definition of 2-cell for a Morse complex, which is the set of the integral lines that originate or converge to a critical point. We discuss here how the algorithms extract the unstable Morse complex. The computation of the stable one is done in a completely symmetric way.

We review here three region growing methods proposed in our previous work. The algorithm in 12 , denoted here as DIS, is based on the elevation values at the TIN vertices, while the one in [11, denoted as GRD, is based on the gradient associated with the triangles. The algorithms sort the vertices according to their elevation and process them in decreasing elevation order. Let $p$ be $p$ the vertex at the highest elevation among unprocessed vertices. The unstable 2-cell associated with $p$, that we denote with $\gamma$, is initialized with all the triangles incident in $p$, which have not yet been assigned to any 2-cell. The 2-cell $\gamma$ is grown by following a predefined criterion until it cannot be extended any more. If the vertex $p$ lies on the boundary of another 2-cell $\gamma^{\prime}$, then $p$ is not a local maximum, and thus $\gamma$ and $\gamma^{\prime}$ are merged.

The region growing criterion, according to which a triangle is added to the 2-cell $\gamma$, is different in the two algorithms. In the DIS algorithm, the current 2-cell $\gamma$ is extended by including the triangle $t=A B C$, adjacent to $\gamma$ along an edge $A B$, if $C$ is the vertex $\mathrm{f} t$ with lowest elevation. Instead, the GRD algorithm incluses $t$ if the gradient of $t$ has the same orientation as the gradient of $t^{\prime}$, where $t^{\prime}=A B D$ is the triangle in $\gamma$ sharing edge $A B$ with $t$.

In the growing algorithm in [20], denoted here as $S T D$, the region growing criterion plays the most important role. Initially, the algorithm labels the highest, 
middle, and lowest vertex in each triangle $t$ as source $(\mathrm{S})$, through $(\mathrm{T})$, and drain (D), respectively. Then, the minima are found as those vertices labeled $D$ in all their incident triangles. For each minimum $m$, the 2-cell $\gamma$ associated with $m$ is initialized with all triangles incident in $m$. A loop follows, in which, at each iteration, the algorithm selects a set of triangles to be included from an edge $e$ of the boundary of the current 2-cell $\gamma$, based on vertex classification.

\subsection{Watershed Algorithms}

The watershed transform has been first introduced in image processing for grayscale images. Several definitions, and related algorithms, exist in the discrete

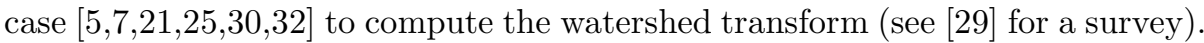
Here, we extended and implemented the two major approaches proposed in the literature, based on simulated immersion [32, and on the discretization of the topographic distance 25, to deal with TINs, and in general triangle meshes in 3D space having a scalar value associated with each vertex. We denote the simulated immersion algorithm for TINs as $W V S$ and as the one using topographic distance as WTD.

\section{Metrics for Comparison}

In this Section, we describe the metrics we use to compare the decompositions of the same TIN produced by different algorithms. Such metrics provide a number between 0 and 1, where 0 indicates that two decompositions $\Gamma_{1}$ and $\Gamma_{2}$ are completely different, and 1 indicates that they are identical [10].

The first metric, called Rand index (RI) 2810, measures the likelihood that a pair of triangles is either in the same region or in different regions in both decompositions. For each pair of triangles, we mark 1 either if they belong to one region in both decompositions, or if they belong to two different regions in both decomposition. Otherwise, we mark 0 . Then, we divide the sum of the values by the number triangle pairs. $R I$ metric assesses the similarity among decompositions even in case in which number of regions is widely different and region matching is problematic.

The second metric is a variant of the Hamming distance defined in 1910]. The general idea is to find a best corresponding 2-cell in decomposition $\Gamma_{1}$ for each 2-cell in decomposition $\Gamma_{2}$, and measure the difference area between the regions. In the original definition, region matching is performed by considering the maximun common area. In our case, we say that two regions (2-cells) are corresponding if they have the same critical point associated. We define as Hamming distance $(H D)$ the ratio between the number of the triangles that are assigned to corresponding 2-cells in the two decompositions, and the total number of triangles. The $H D$ metric heavily relies on region matching, and is strongly affected even in case of a few mismatches. 


\section{Experimental Results}

We have applied the algorithms presented in Section 4 both on synthetic and real datasets, and compared the Morse decompositions by using the metrics described in Section 5. Real data are affected by sampling errors, and, in most cases, contain flat areas (plains, lakes etc.); thus, real TINs do not correspond to Morse functions.

\subsection{Input Data}

We have experimented on three groups of real terrain data sets. The datasets of the first group are TINs over subsets of data from the CGIAR-CSI GeoPortal, [1. Such TINs are composed of vertices distributed on a regular square grid and isosceles right triangles all of the same size. In the following, we refer to them as regular TINs. These TINs are available on the Aim@Shape repository [2]. The TINs of second group are extracted from a regular data set but triangulated in a nested triangle structure, called a hierarchy of diamonds [34. The resulting TINs are crack-free triangle meshes extracted from the hierarchy and are composed by isosceles right triangles of different sizes. In the following, we refer to them as diamonds TINs. The third group contains irregular triangle meshes, that we call irregular TINs.

Table 1. Datasets used in the experiments

\begin{tabular}{|c|c|c|c|c|}
\hline & Name & \#Vertices & \#Triangles & ePL \\
\hline \multirow{6}{*}{ 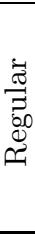 } & MontBlanc & 14400 & 28322 & reducible \\
\hline & MontBlanc-ePL & 14394 & 28310 & yes \\
\hline & Elba & 36036 & 70220 & reducible \\
\hline & Elba-ePL & 35365 & 69059 & yes \\
\hline & Monviso & 160000 & 318402 & reducible \\
\hline & Monviso-ePL & 159606 & 317623 & yes \\
\hline \multirow{7}{*}{ 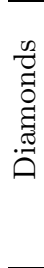 } & San Bern. & 8022 & 15610 & reducible \\
\hline & San Bern-ePL & 7854 & 15286 & yes \\
\hline & Marcy0003 & 214287 & 427242 & no \\
\hline & Marcy003 & 14089 & 27502 & reducible \\
\hline & Marcy003-ePL & 13722 & 26867 & yes \\
\hline & Hawaii0003 & 120456 & 240840 & no \\
\hline & Hawaii001 & 15196 & 29599 & no \\
\hline \multirow{6}{*}{ 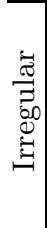 } & Vobbia & 6095 & 11838 & yes \\
\hline & Zqn & 57865 & 114765 & reducible \\
\hline & Zqn-ePL & 57816 & 114695 & yes \\
\hline & Dolomiti & 10004 & 19800 & reducible \\
\hline & Dolomiti-ePL & 9983 & 19759 & yes \\
\hline & Marcy50 & 50004 & 99345 & no \\
\hline
\end{tabular}




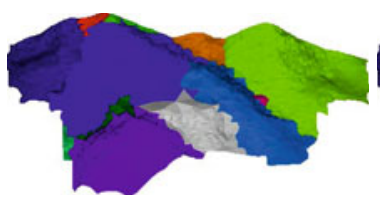

(a)

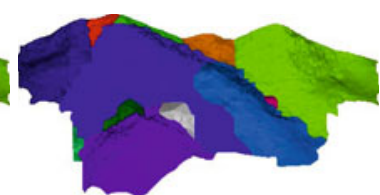

(b)

Fig. 1. Unstable decompositions of Vobbia, produced by TKH (a) and BBD (b)

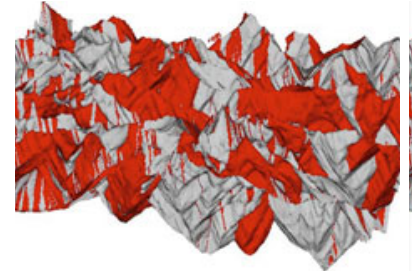

(a)

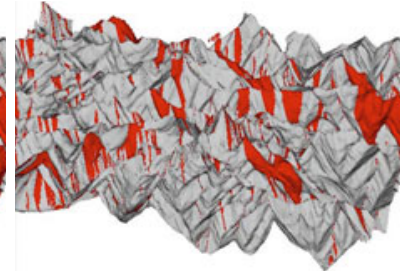

(b)

Fig. 2. Comparison between stable decompositions of Monviso (a) and Monviso ePL (b), produced by WVS and WTD. Red areas represent mismatching

We classify those meshes into (i) ePL-Morse meshes, i.e., meshes that have no critical flat edges, (ii) meshes which can be reduced to ePL-Morse meshes, and (iii) meshes not reducible to ePL-Morse ones. For meshes that are reducible to ePL-Morse meshes, we consider both the original mesh and the mesh obtained by replacing each critical flat component with a component that is not flat, but has the same critical features. We denote those meshes with -ePL suffix. Table 1 shows the list of our test TINs. Figure 1 shows unstable decompositions of Vobbia TIN produced by TKH (a) and BBD (b).

\subsection{Results}

On ePL-meshes, all algorithms find the same number of regions in both the stable and the unstable decompositions. We have compared the algorithms pairwise, according to the Rand-Index (RI) and Hamming-Distance (HD) metrics. RI has, for each pair of algorithms, very high values, almost always in the top $2 \%$. For regular or diamonds ePL-meshes, $\mathrm{HD}$ is in the range $80 \%-99 \%$. In particular, TKH, WVS and STD algorithmd give very similar decompositions (HD is higher than 95\%). As soon as the shape of triangles become less regular, decompositions computed by TKH, WVS and STD algorithms start diverging, and HD becomes as low as 50\%. GRD, WTD and BBD, that simulate the differentiability by considering in some way the slope of triangles, give always pretty similar results, with HD from $80 \%$ to $90 \%$.

Decompositions obtained from non-ePL-meshes can be quite different, even if the number of critical edges is small. Figure 2 shows mismatching between WVS and WTD for a non-ePL-meshe and its ePL version. When we have a 
large number of critical edges, or wide flat areas, the mesh cannot be reduced to ePL-Morse, and both metrics show very low values. For the Hawaii dataset $\mathrm{RI}$ is in the range $57-82 \%$, and $\mathrm{HD}$ is in the range $21-43 \%$, mainly due to the fact that the dataset is an island, and the boundary is a minimum composed by a long chain of edges. On Marcy dataset, RI is the range 89-95\%, and HD is in the range $20-42 \%$, due to a large flat area representing a river with small islands inside. For full details on the metrics comparing decomposition algorithm pairwise, please refer to 33 .

\section{Concluding Remarks}

We have considered the problem of computing the morphology of triangulated terrains and we have presented and analyzed the various approaches for computing it. In Table 2, we summarize the main properties of the algorithms. GRD and WVS sort vertices and detect maxima and minima during computation, while STD, WTD, TKH and BBD use Banchoff's characterization (see Section 3) to find maxima and minima in a preprocessing step. STD, WVS and TKH are fully discrete and consider only the elevation values at the vertices. Decompositions of regular grid are similar for all fully discrete algorithms. GRD, WTD and BBD, take edge slope into account, but simulate the differentiability in various ways. Decompositions resulting from regular TINs are quite similar. Resulting decompositions on irregular TINs are different, but GRD, WTD and BBD that simulate the differentiability (even if in different ways), give results more similar among them than STD, WVS and TKH algorithms.

GRD and WVS can deal with higher dimensional data. WTD can be extended quite easily, while STD in higher dimension requires handling of a large number of local configurations. BBS and TKH are not extensible to higher dimensions, since they compute separatrix lines, while in higher dimensions we should compute hypersurfaces. See [33] for further details.

Table 2. Main properties of the algorithms

\begin{tabular}{c|c|c|c|} 
& $\begin{array}{c}\text { Extraction of } \\
\text { critical points }\end{array}$ & $\begin{array}{c}\text { Simulation of } \\
\text { Differentiability }\end{array}$ & $\begin{array}{c}\text { Scale easily to } \\
\text { higher dimension }\end{array}$ \\
\hline GRD & Sort vertices & Gradient & Yes \\
\hline STD & Banchoff & Fully Discrete & No \\
\hline WTD & Banchoff & Top. Distance & No \\
\hline WVS & Sort vertices & Fully Discrete & Yes \\
\hline TKH & Sort vertices & Fully Discrete & No \\
\hline BBD & Sort vertices & Diff. Quotient & No \\
\hline
\end{tabular}


The size of a morphological representation can be large for common models, which may consist of several millions of triangles. To deal with problem, we have been focusing on a multiresolution representation of the terrain morphology (see [13]), that allows concentrating on the areas of interest and producing approximate representations of the morphology at uniform and variable resolution. In our current work, we are developing a multiresolution model that combines the geometrical and morphological representations, where the morphological representation as an index on the geometrical model [14].

\section{References}

1. Reuter, J.A.H., Nelson, A., Guevara, E.: Hole-filled seamless srtm data v4, international centre for tropical agriculture, ciat (2008), http://srtm.csi.cgiar.org

2. AIM@SHAPE. Shape Repository (2006), http://shapes.aimatshape.net

3. Bajaj, C.L., Shikore, D.R.: Topology preserving data simplification with error bounds. Computers and Graphics 22(1), 3-12 (1998)

4. Banchoff, T.: Critical points and curvature for embedded polyhedral surfaces. American Mathematical Monthly 77(5), 475-485 (1970)

5. Beucher, S., Lantuejoul, C.: Use of watersheds in contour detection. In: Int. Workshop on Image Processing: Real-Time Edge and Motion Detection/Estimation (1979)

6. Biasotti, S., De Floriani, L., Falcidieno, B., Frosini, P., Giorgi, D., Landi, C., Papaleo, L., Spagnuolo, M.: Describing shapes by geometrical-topological properties of real functions. ACM Computing Surveys 40(4), 1-87 (2008)

7. Bieniek, A., Moga, A.: A connected component approach to the watershed segmentation. In: Mathematical Morphology and its Application to Image and Signal Processing, pp. 215-222. Kluwer Acad. Publ., Dordrecht (1998)

8. Bremer, P.-T., Edelsbrunner, H., Hamann, B., Pascucci, V.: A topological hierarchy for functions on triangulated surfaces. IEEE Transactions on Visualization and Computer Graphics 10(4), 385-396 (2004)

9. Cazals, F., Chazal, F., Lewiner, T.: Molecular shape analysis based upon the MorseSmale complex and the connolly function. In: SCG 2003: Proc. 19th annual symposium on Computational geometry, pp. 351-360. ACM Press, New York (2003)

10. Chen, X., Golovinskiy, A., Funkhouser, T.: A benchmark for 3D mesh segmentation. ACM Transactions on Graphics (Proc. SIGGRAPH) 28(3) (2009)

11. Danovaro, E., De Floriani, L., Magillo, P., Mesmoudi, M.M., Puppo, E.: Morphology-driven simplification and multi-resolution modeling of terrains. In: Proc. 11th ACMGIS, pp. 63-70. ACM Press, New York (2003)

12. Danovaro, E., De Floriani, L., Mesmoudi, M.M.: Topological analysis and characterization of discrete scalar fields. In: Asano, T., Klette, R., Ronse, C. (eds.) Geometry, Morphology, and Computational Imaging. LNCS, vol. 2616, pp. 386402. Springer, Heidelberg (2003)

13. Danovaro, E., De Floriani, L., Vitali, M., Magillo, P.: Multi-scale dual Morse complexes for representing terrain morphology. In: Proc. 15th ACMGIS. ACM Press, New York (2007)

14. Danovaro, E., Floriani, L.D., Magillo, P., Vitali, M.: Multiresolution Morse triangulations. In: Proc. Symposium of Solid and Physical Modeling (2010) 
15. De Floriani, L., Magillo, P., Vitali, M.: Modeling and generalization of discrete Morse terrain decompositions. In: Proc. 20th Int. Conf. on Pattern Recognition, ICPR 2010, pp. 999-1002. IEEE Computer Society, Los Alamitos (2010)

16. Edelsbrunner, H., Harer, J., Natarajan, V., Pascucci, V.: Morse-Smale complexes for piecewise linear 3-manifolds. In: Proc. 19th ACM Symposium on Computational Geometry, pp. 361-370 (2003)

17. Edelsbrunner, H., Harer, J., Zomorodian, A.: Hierarchical Morse complexes for piecewise linear 2-manifolds. In: Proc. 17th ACM Symposium on Computational Geometry, pp. 70-79. ACM Press, New York (2001)

18. Forman, R.: Morse theory for cell complexes. Adv. in Math. 134, 90-145 (1998)

19. Huang, Q., Dom, B.: Quantitative methods of evaluating image segmentation. In: ICIP 1995: Proc. Int. Conf. on Image Processing, vol. 3, p. 3053. IEEE Computer Society, Washington, DC, USA (1995)

20. Magillo, P., Danovaro, E., De Floriani, L., Papaleo, L., Vitali, M.: A discrete approach to compute terrain morphology. Computer Vision and Computer Graphics. Theory and Applications 21, 13-26 (2009)

21. Meijster, A., Roerdink, J.: Computation of watersheds based on parallel graph algorithms. In: Mathematical Morphology and its Application to Image Segmentation, pp. 305-312. Kluwer, Dordrecht (1996)

22. Mesmoudi, M., Danovaro, E., De Floriani, L., Port, U.: Surface segmentation through concentrated curvature. In: Proc. ICIAP, pp. 671-676 (2007)

23. Mesmoudi, M., Floriani, L.D., Magillo, P.: Discrete distortion for surface meshes. In: Proc. ICIAP (2009)

24. Mesmoudi, M., Floriani, L.D., Magillo, P.: A geometric approach to curvature estimation on triangulated 3D shapes. In: Int. Conf. on Computer Graphics Theory and Applications (GRAPP), pp. 90-95 (2010)

25. Meyer, F.: Topographic distance and watershed lines. Signal Processing 38, 113125 (1994)

26. Milnor, J.: Morse Theory. Princeton University Press, Princeton (1963)

27. Pascucci, V.: Topology diagrams of scalar fields in scientific visualization. In: Topological Data Structures for Surfaces, pp. 121-129. John Wiley and Sons Ltd, Chichester (2004)

28. Rand, W.: Objective criteria for the evaluation of clusterings methods. Journal of the American Statistical Association 66(336), 846-850 (1971)

29. Roerdink, J., Meijster, A.: The watershed transform: definitions, algorithms, and parallelization strategies. Fundamenta Informaticae 41, 187-228 (2000)

30. Stoev, S.L., Strasser, W.: Extracting regions of interest applying a local watershed transformation. In: Proc. IEEE Visualization 2000, pp. 21-28. IEEE Computer Society, Los Alamitos (2000)

31. Takahashi, S., Ikeda, T., Kunii, T.L., Ueda, M.: Algorithms for extracting correct critical points and constructing topological graphs from discrete geographic elevation data. Computer Graphics Forum 14(3), 181-192 (1995)

32. Vincent, L., Soile, P.: Watershed in digital spaces: an efficient algorithm based on immersion simulation. IEEE Transactions on Pattern Analysis and Machine Intelligence 13(6), 583-598 (1991)

33. Vitali, M.: Morse Decomposition of Geometric Meshes with Applications. PhD thesis, Computer Science Dept., University of Genova, Italy (2010)

34. Weiss, K., De Floriani, L.: Sparse terrain pyramids. In: Proc. ACM SIGSPATIAL GIS, pp. 115-124. ACM Press, New York (2008) 